J. JOHN, E. GRAVEL, A. HAGÈGE, H. LI, T. GACOIN, E. DORIS* (XIAMEN UNIVERSITY, P. R. OF CHINA; ÉCOLE POLYTECHNiQUe, PALAiseAu, CEA, IBITECS, Gif-SUR-YVETTE, CEA, IBEB, BAGNOLS-SUR-CÈZE AND CEA, IBEB, SAINT PAUL-LES-DURANCE, FRANCE) Catalytic Oxidation of Silanes by Carbon Nanotube-Gold Nanohybrids Angew. Chem. Int. Ed. 2011, 50, 7533-7536.

\title{
Silane Oxidation Catalyzed by Carbon Nanotube-Gold Nanohybrids
}

Polymer-Supported Synthesis

Key words

carbon nanotubes

oxidation

heterogeneous catalysis

layer-by-layer

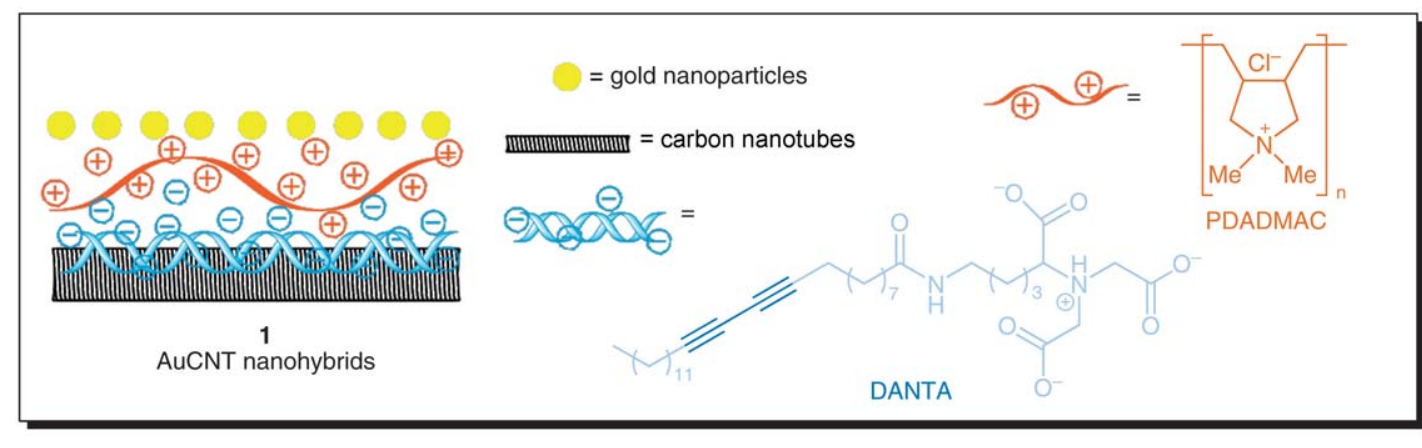

Results: $\underset{2 \mathrm{a}-\mathrm{j}(0.2 \mathrm{mmol})}{\mathrm{R}^{1} \mathrm{Si}\left(\mathrm{R}^{2}\right)_{2} \mathrm{H}}+n \mathrm{H}_{2} \mathrm{O} \stackrel{\text { AuCNTs }(0.1 \mathrm{~mol} \%) \text {, air }}{\underset{\text { THF, r.t., time }}{\longrightarrow}} \underset{\substack{3 \mathrm{a}-\mathrm{j} \\ \mathrm{R}}}{\mathrm{R}} \mathrm{Si}\left(\mathrm{R}^{2}\right)_{2} \mathrm{OH}+n \mathrm{H}_{2}$<smiles>C[Si](O)([Mg])c1ccccc1</smiles>
$45 \mathrm{~min}, 98 \%$ yield<smiles>CC[Si](O)(CC)CC</smiles>

$3 \mathrm{~b}$ 15 min, $99 \%$ yield

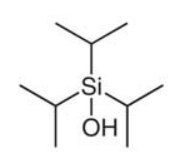

$3 c$ $60 \mathrm{~min}, 93 \%$ yield<smiles>O[Si](O)(c1ccccc1)c1ccccc1</smiles>

3d $30 \mathrm{~min}, 99 \%$ yield

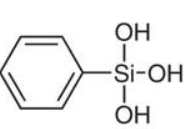

$3 e$ $90 \mathrm{~min}, 97 \%$ yield<smiles>O[Si](c1ccccc1)(c1ccccc1)c1ccccc1</smiles>

$3 f$<smiles>COc1ccc(C#C[Si](C)(C)O)cc1</smiles>

$3 \mathrm{~g}$ $240 \mathrm{~min}, 96 \%$ yield

$180 \mathrm{~min}, 98 \%$ yield

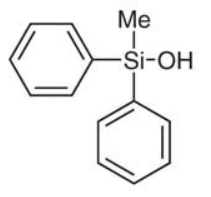

3h $120 \mathrm{~min}, 96 \%$ yield<smiles>C[Si](O)(Cc1ccccc1)O[Na]</smiles>

$3 \mathbf{i}$ $45 \mathrm{~min}, 98 \%$ yield

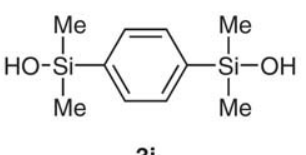

$3 \mathbf{j}$

$180 \mathrm{~min}, 96 \%$ yield
Significance: The gold nanohybrid on multiwalled carbon nanotubes 1 (AuCNT nanohybrids) was prepared by layer-by-layer ( $L B L$ ) assembly of amphiphilic nitrilotriacetic acid diyne lipids (DANTA), cationic poly(diallyldimethylammonium chloride) (PDA-DMAC), and colloidal nanoparticles (AuNPs). The AuCNT-catalyzed aerobic oxidation of silanes $(\mathbf{2} \mathbf{a}-\mathbf{j})$ was carried out in THF to give the corresponding silanols (3a-j) in 93-99\% yields.
Comment: The hydrophobic portion of DANTA was adsorbed on the nanotubes and photopolymerized by UV irradiation at $254 \mathrm{~nm}$. Carbon nanotube-gold nanohybrid $\mathbf{1}$ was characterized by TEM, GC-MS, ICP-MS, and XPS analyses. The catalyst was readily recovered by centrifugation and reused five times without significant loss of catalytic activity. 